\title{
Asymptotic Performance Analysis of Time-Frequency-Domain Spread MC DS-CDMA Systems Employing MMSE Multiuser Detection
}

\author{
Peng Pan and Youguang Zhang \\ School of Electronic and Information Engineering \\ Beihang University \\ Beijing 100083, China \\ Email: pp07v,zhangyouguang@vip.sina.com
}

\author{
Lie-Liang Yang \\ School of ECS, University of Southampton \\ SO17 1BJ, United Kingdom \\ Tel: 0044-(0)23-8059 3364, Email: 1ly@ecs.soton.ac.uk \\ http://www-mobile.ecs.soton.ac.uk
}

\begin{abstract}
In this contribution the asymptotic signal-to-interferenceplus-noise ratio (SINR) performance of multicarrier direct-sequence code-division multiple-access systems employing time-frequency-domain spreading, i.e., of the TF/MC DS-CDMA systems, is studied, when separate minimum mean-square error multiuser detection (MMSE-MUD) is considered. The separate MMSE-MUD detects signals first in the time (T)-domain and then in the frequency (F)-domain. Based on random matrix theory, closed-form expressions for the asymptotic SINR of the TF/MC DS-CDMA systems using separate MMSE-MUD is derived, when communicating over additive white Gaussian noise (AWGN) channels. The closed-form expressions show that the asymptotic SINR performance is only depended on the T- and F-domain user load factors as well as noise variance. Hence, they are beneficial to evaluation. Furthermore, our simulation and numerical results show that in most cases the asymptotic SINR can provide a good approximation to the SINR achieved by realistic TF/MC DS-CDMA systems employing separate MMSE-MUD.
\end{abstract}

\section{INTRODUCTION}

Multicarrier techniques have been recognized the promising techniques for broadband wireless communications [1-3]. Recently, multicarrier DS-CDMA employing both time (T)-domain and frequency (F)-domain spreading, which is referred to as the TF/MC DS-CDMA, has been proposed for broadband wireless communications, owning to its high-flexibility of system design and reconfiguration [4,5]. Due to the employment of two dimensions of spreading, MUD in the TF/MC DS-CDMA systems is capable of achieving a good trade-off between complexity and performance $[3,4]$. The MUD in the TF/MC DSCDMA systems can be carried out separately in two steps, first in the T-domain and, then, in the F-domain $[4,5]$. The complexity of the separate MUD can be significantly lower than that of the conventional (or joint) MUD, which carries out the MUD jointly in the T-domain and F-domain. In this contribution, we analyze the asymptotic SINR performance of the separate MMSE-MUD for the TF/MC DS-CDMA systems employ random spreading sequences, when communicating over AWGN channels. Furthermore, we illustrate the effectiveness of the analytical results obtained under the asymptotic conditions by comparing them with the corresponding results obtained by simulations.

Note that, in this contribution asymptotic means that both the number of users, say $K$, supported by a TF/MC DS-CDMA system and the spreading factor, say $\mathcal{N}$, tend to infinity, while the ratio $\beta=K / \mathcal{N}$ retains constant.

With the aid of random matrix theory [6], asymptotic SINR performance of MUDs has been investigated in a number of references, such as, e.g., in [7-9, 11] for CDMA and in [12] for multi-antenna MIMO systems. As the studies in [7-10] shown, in DS-CDMA systems the SINR of the MMSE-MUD converges to a deterministic expression as the number of users and spreading factor tend to infinity with their ratio fixed, when communicating over Gaussian channels [7,8] or over multipath fading channels [9]. Similarly, the asymptotic performance of multicarrier CDMA systems using MUD has been studied in [11], which also results in closed-form expressions of SINR. Furthermore, it can be shown that the closed-form expressions obtained from the asymptotic analysis are effective for practical application, since in most cases they provide results that are close to their practical counterparts, as seen in [6] and also in Section IV of this paper.

In this contribution, we derive the asymptotic SINR expressions of the TF/MC DS-CDMA systems using separate MMSE-MUD. Both Gaussian approximation method (GAM) and accurate method (ACM) assisted MMSE-MUDs are considered. For the GAM-assisted MMSEMUD the residual multiuser interference (MUI) after the T-domain MMSE-MUD is approximated as independent Gaussian random variables, when carrying out the following F-domain MMSE-MUD. By contrast, the ACM-assisted MMSE-MUD treats the residual MUI from the T-domain MMSE-MUD as interference, which is further suppressed by the F-domain MMSE-MUD. Our studies show that the ACM-assisted MMSE-MUD usually outperforms the GAM-assisted MMSE-MUD, when considering their achievable SINR performance.

\section{TF/MC DS-CDMA SySTEMS EMPLOYING SEPARATE MMSE-MUD}

A. Signal Model

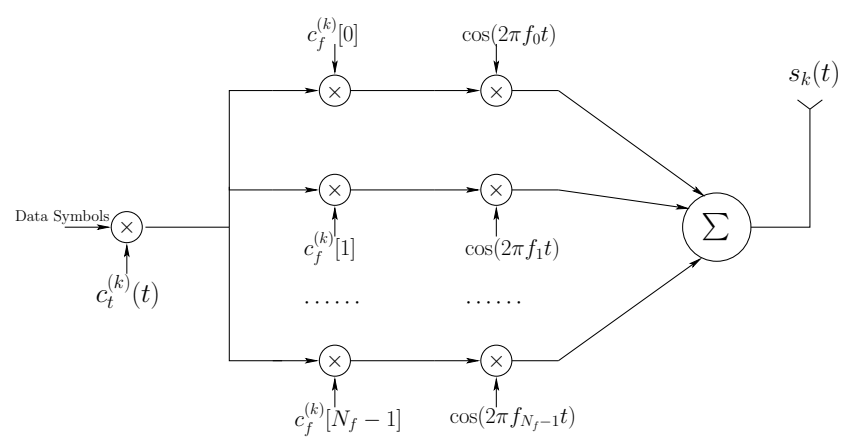

Fig. 1. Transmitter model of MC DS-CDMA using both time- and frequencydomain spreading.

The transmitter schematic block diagram for the $k$ th user supported by the TF/MC DS-CDMA is shown in Fig.1, which is the same as that in [13]. For convenience, the main parameters used are summarized as below:

- $N_{f}$ : Number of orthogonal subcarriers or F-domain spreading factor;

- $N_{t}$ : T-domain spreading factor;

- $\mathcal{N}=N_{f} N_{t}$ : Total spreading factor of TF/MC DS-CDMA;

- $N / M$ : Number of T-domain/F-domain spreading sequences assigned to the active users supported by the TF/MC DS-CDMA system;

- $K$ : Total number of active users, which is assumed to be $K=$ $M N$; 
- $\beta_{t}, \beta_{f}, \beta$ : T-domain load factor, F-domain load factor and total system load factor, respectively, where $\beta_{t}=N / N_{t}, \beta_{f}=$ $M / N_{f}$ and $\beta=\beta_{t} \beta_{f}=M N / \mathcal{N}$.

As shown in Fig.1, the data symbols transmitted by the $k$ th user are first spread in the T-domain using a T-domain signature waveform $c_{t}^{(k)}(t)$ formed from a T-domain spreading sequence $\boldsymbol{c}_{t}^{(k)}$ assigned to the $k$ th user. Then, the T-domain spreading signal is copied to $N_{f}$ parallel sub-streams, each of which is multiplied by a chip of the Fdomain spreading sequence $\boldsymbol{c}_{f}^{(k)}$ to achieve the F-domain spreading. Note that, in order to make the $N_{f}$ subcarriers orthogonal, the Fdomain spacing between two adjacent subcarriers is assumed $1 / T_{c}$, where $T_{c}$ denotes the chip-duration of the T-domain signature waveform.

For the TF/MC DS-CDMA system considered in this paper, the following assumptions are applied.

A1. $K$ number of user signals are synchronously transmitted to the base-station (BS);

A2. Binary phase-shift keying (BPSK) data modulation with $P\left(b_{k}=\right.$ $+1)=P\left(b_{k}=-1\right)=0.5$;

A3. Perfect power-control makes the received power from each of the users the same;

A4. Random spreading sequences, whose elements are iid circularly symmetric complex random variables with zero mean and variance $1 / N_{t}$ for T-domain spreading sequences while variance $1 / N_{f}$ for F-domain spreading sequences. Hence, the T-domain and F-domain sequences are normalized to $\left\|\boldsymbol{c}_{t}^{(k)}\right\|^{2}=1$ and $\left\|\boldsymbol{c}_{f}\right\|^{2}=1$.

Based on above assumptions, the observation vector obtained from the $N_{f}$ filters matching to the chip-waveform of the DS signature waveform can be expressed as [13]

$$
\boldsymbol{y}=\sum_{k=1}^{K}\left(\boldsymbol{c}_{f}^{(k)} \otimes \boldsymbol{c}_{t}^{(k)}\right) b_{k}+\boldsymbol{n}
$$

where $\otimes$ denotes Kronecker product operation, while $\boldsymbol{n}$ is a $N_{f} N_{t^{-}}$ length circularly symmetric complex Gaussian noise vector that is distributed with zero mean and a covariance matrix $\sigma^{2} \boldsymbol{I}_{N_{f} N_{t}}$, where $\sigma^{2}=1 / \mathrm{SNR}$ and SNR is the signal-to-noise ratio (SNR). Since there is no interference between any two subcarriers, the $N_{f} N_{t}$ length observation vector $\boldsymbol{y}$ can be divided into $N_{f}$ sub-vectors, each corresponds to one subcarrier:

$$
\begin{gathered}
\boldsymbol{y}=\left[\boldsymbol{y}_{1}^{T}, \boldsymbol{y}_{2}^{T}, \cdots, \boldsymbol{y}_{N_{f}}^{T}\right]^{T} \\
\boldsymbol{y}_{v}=\left[y_{v 1}, y_{v 2}, \cdots, y_{v N_{t}}\right]^{T}
\end{gathered}
$$

where $\boldsymbol{y}_{v}$ is a $N_{t}$-length vector containing $N_{t}$ observation samples of the $v$ th subcarrier within one symbol duration, and $y_{v n}$ is the $n$th sample from the $v$ th subcarrier.

The $K=M N$ number of active users are assigned their T-domain and F-domain spreading sequences according to the following rules: The $k$ th user is assigned the T-domain spreading sequence $\boldsymbol{c}_{t}^{(n)}$, if $n=(k-\lfloor(k-1) / N\rfloor N)$, where the operation $\lfloor x\rfloor$ returns the largest integer not exceeding $x$; The $k$ th user is assigned the F-domain spreading sequence $\boldsymbol{c}_{f}^{(m)}$, if $m=\lfloor(k-1) / N\rfloor+1$. Under this sequence assignment scheme, we can show that (1) can be rewritten as

$$
\begin{aligned}
\boldsymbol{y} & =\sum_{m=1}^{M} \sum_{n=1}^{N}\left(\boldsymbol{c}_{f}^{(m)} \otimes \boldsymbol{c}_{t}^{(n)}\right) b_{n m}+\boldsymbol{n} \\
& =\left(\boldsymbol{C}_{f} \otimes \boldsymbol{C}_{t}\right) \boldsymbol{b}+\boldsymbol{n}=\boldsymbol{C} \boldsymbol{b}+\boldsymbol{n}
\end{aligned}
$$

where $\boldsymbol{C}_{f}=\left[\boldsymbol{c}_{f}^{(1)}, \cdots, \boldsymbol{c}_{f}^{(M)}\right], \boldsymbol{C}_{t}=\left[\boldsymbol{c}_{t}^{(1)}, \cdots, \boldsymbol{c}_{t}^{(N)}\right], \boldsymbol{b}=$ $\left[b_{1}, b_{2}, \ldots, b_{K}\right]^{T}$ and $\boldsymbol{C}=\boldsymbol{C}_{f} \otimes \boldsymbol{C}_{t}$.

According to the spreading sequence assignment scheme and (3), we can see that the $K$ users can be divided into $M$ groups with each group containing $N$ users. Users in one group share the same F-domain spreading sequence but are distinguished by different Tdomain spreading sequences. By contrast, the $M$ groups can be distinguished by the $M$ number of unique F-domain spreading sequences. Let us below consider the principles of the separate MMSE-MUD.

\section{B. Separate MMSE-MUD}

The T-domain MMSE-MUD is implemented associated with each of the $N_{f}$ subcarriers. Specifically, for the $v$ th subcarrier, the observation vector $\boldsymbol{y}_{v}$ can be expressed as

$$
\boldsymbol{y}_{v}=\boldsymbol{C}_{t} \boldsymbol{x}+\boldsymbol{n}_{v}
$$

where $\boldsymbol{n}_{v}$ is $N_{t}$-length and contains the noise samples from the $v$ th subcarrier, and the $n$th element of $x$ is given by $x_{n}=$ $\sum_{m=1}^{M} c_{f v}^{(m)} b_{n m}$, where each pair of $(m, n)$ determines a corresponding index $k$, as specified in the last subsection.

After correlation operation, the sufficient statistics of $x$ can be expressed as

$$
\tilde{\boldsymbol{y}}_{v}=\boldsymbol{R}_{t} \boldsymbol{x}+\tilde{\boldsymbol{n}}_{v}, v=1,2, \ldots, N_{f}
$$

where $\boldsymbol{R}_{t}=\boldsymbol{C}_{t}^{H} \boldsymbol{C}_{t}$ and $\tilde{\boldsymbol{n}}_{v}=\boldsymbol{C}_{t}^{H} \boldsymbol{n}_{v}$. Based on (5), it can be readily shown that the T-domain MMSE-MUD can be implemented by multiplying $\tilde{\boldsymbol{y}}_{v}$ with a weight matrix $\boldsymbol{W}_{t}=\left(\boldsymbol{R}_{t}+N_{f} \sigma^{2} \boldsymbol{I}_{N} / M\right)^{-1}[14]$, which is independent of the index $v$. The above MMSE solution minimizes the MSE in terms of each individual subcarrier. However, it does not result in maximization of the overall SINR when considering together the $N_{f}$ subcarriers. After some tests, we find that a better solution for the $v$ th subcarrier, which results in higher overall SINR than that achieved by using the weight matrix $\boldsymbol{W}_{t}=\left(\boldsymbol{R}_{t}+\right.$ $\left.N_{f} \sigma^{2} \boldsymbol{I}_{N} / M\right)^{-1}$, is $\left(\boldsymbol{R}_{t}+\sigma^{2} \boldsymbol{I}_{N}\right)^{-1}$. Therefore, in this contribution we use the T-domain weight matrix $\boldsymbol{W}_{t}=\left(\boldsymbol{R}_{t}+\sigma^{2} \boldsymbol{I}_{N}\right)^{-1}$ instead of the one derived directly from (5).

Let $\boldsymbol{Q}=\left(\boldsymbol{R}_{t}+\sigma^{2} \boldsymbol{I}_{N}\right)^{-1} \boldsymbol{R}_{t}$ and $\boldsymbol{P}=\left(\boldsymbol{R}_{t}+\sigma^{2} \boldsymbol{I}_{N}\right)^{-1} \boldsymbol{C}_{t}^{H}$, the soft output of the T-domain MMSE-MUD at the $v$ th subcarrier can be written as

$$
z_{v}=Q x+P n_{v}
$$

Let the first user belonging to the first user group be the desired user. Let $\boldsymbol{r}_{1}=\left[z_{11}, z_{21}, \cdots, z_{N_{f} 1}\right]^{T}$ contain all the T-domain MMSEMUD's outputs corresponding to the first user group. Then, $\boldsymbol{r}_{1}$ can be expressed as

$$
\boldsymbol{r}_{1}=q_{11} \boldsymbol{C}_{f} \boldsymbol{b}_{1}+\sum_{n=2}^{N} q_{1 n} \boldsymbol{C}_{f} \boldsymbol{b}_{n}+\boldsymbol{n}^{\prime}
$$

where the second term at the righthand side is the MUI caused by the other user groups and the third term is the Gaussian noise distributed with zero mean and a covariance matrix $\sigma^{2} \sum_{n=1}^{N_{t}}\left|p_{1 n}\right|^{2} \boldsymbol{I}_{N_{f}}$. Finally, $p_{i j}$ and $q_{i j}$ represent the $(i, j)$ th entries of $\boldsymbol{P}$ and $\boldsymbol{Q}$, respectively.

Let us now turn to consider the F-domain MMSE-MUD, for which two methods are considered. The first one is the Gaussian approximation method (GAM), which approximates the residual MUI from the T-domain MMSE-MUD as independent additive Gaussian noise. By contrast, the second one is the accurate method (ACM), which further suppresses the residual MUI from the T-domain MMSE-MUD. In the context of the GAM, the F-domain weight vector in MMSE sense for detecting $b_{1}$ of the first user can be expressed as

$$
\boldsymbol{w}_{g a m}=\left(\boldsymbol{C}_{f} \boldsymbol{C}_{f}^{H}+\frac{1}{\gamma_{t}} \boldsymbol{I}_{N_{f}}\right)^{-1} \boldsymbol{c}_{f}^{(1)}
$$

where $\gamma_{t}=\left|q_{11}\right|^{2} /\left(\sum_{n=2}^{N}\left|q_{1 n}\right|^{2}+\sigma^{2} \sum_{n=1}^{N_{t}}\left|p_{1 n}\right|^{2}\right)$ is the T-domain MMSE-MUD's output SINR, which is in fact equal to

$$
\gamma_{t}=\left(\boldsymbol{c}_{t}^{(1)}\right)^{H}\left(\boldsymbol{C}_{t 1} \boldsymbol{C}_{t 1}^{H}+\sigma^{2} \boldsymbol{I}_{N_{t}}\right)^{-1} \boldsymbol{c}_{t}^{(1)}
$$


where $\boldsymbol{C}_{t 1}$ is obtained from $\boldsymbol{C}_{t}$ by removing its first column.

By contrast, for the ACM, based on (7) we can show that the Fdomain weight vector in MMSE sense can be expressed as

$$
\boldsymbol{w}_{a c m}=\left(\sum_{n=1}^{N}\left|q_{1 n}\right|^{2} \boldsymbol{C}_{f} \boldsymbol{C}_{f}^{H}+\sum_{n=1}^{N_{t}}\left|p_{1 n}\right|^{2} \sigma^{2} \boldsymbol{I}_{N_{f}}\right)^{-1} \boldsymbol{c}_{f}^{(1)}
$$

Finally, the decision variable for detection of $b_{1}$ can be formed as

$$
\hat{b}_{1}=\boldsymbol{w}_{g a m}^{H} \boldsymbol{r}_{1} \text { or } \hat{b}_{1}=\boldsymbol{w}_{a c m}^{H} \boldsymbol{r}_{1}
$$

for the GAM or ACM, respectively.

After both the T-domain and F-domain MMSE-MUDs, it can be shown that the final SINR corresponding to the GAM is given by

$$
\gamma_{g a m}=\left(\boldsymbol{c}_{f}^{(1)}\right)^{H}\left(\boldsymbol{C}_{f 1} \boldsymbol{C}_{f 1}^{H}+\frac{1}{\gamma_{t}} \boldsymbol{I}_{N_{f}}\right)^{-1} \boldsymbol{c}_{f}^{(1)}
$$

where $\boldsymbol{C}_{f 1}$ is formed from $\boldsymbol{C}_{f}$ by removing its first column. The final SINR corresponding to the ACM is given by

$$
\begin{array}{r}
\gamma_{a c m}=\left(\boldsymbol{c}_{f}^{(1)}\right)^{H}\left(\boldsymbol{C}_{f 1} \boldsymbol{C}_{f 1}^{H}+\sum_{n=2}^{N} \frac{\left|q_{1 n}\right|^{2}}{\left|q_{11}\right|^{2}} \boldsymbol{C}_{f} \boldsymbol{C}_{f}^{H}\right. \\
\left.+\sum_{n=1}^{N_{t}} \frac{\left|p_{1 n}\right|^{2}}{\left|q_{11}\right|^{2}} \sigma^{2} \boldsymbol{I}_{N_{f}}\right)^{-1} \boldsymbol{c}_{f}^{(1)}
\end{array}
$$

Let us now analyse the asymptotic SINR performance of the TF/MC DS-CDMA systems based on (12) and (13).

\section{DERIVATION OF ASYMPTOTIC SINR}

It is well-known that the output SINR after the MMSE-MUD can be expressed in the form of [14]

$$
\gamma_{k}=P_{k} \boldsymbol{s}_{k}^{H}\left(\boldsymbol{S}_{k} \boldsymbol{D}_{k} \boldsymbol{S}_{k}^{H}+\sigma^{2} \boldsymbol{I}\right)^{-1} \boldsymbol{s}_{k}
$$

where $\boldsymbol{s}_{k}$ is the $k$ th user's spreading sequence of length $\mathcal{N}, \quad \boldsymbol{S}_{k}=\left[\boldsymbol{s}_{1}, \cdots, \boldsymbol{s}_{k-1}, \boldsymbol{s}_{k+1}, \cdots, \boldsymbol{s}_{K}\right]$ and $\boldsymbol{D}_{k}=$ $\operatorname{diag}\left(P_{1}, \cdots, P_{k-1}, P_{k+1}, \cdots, P_{K}\right)$, where $P_{k}$ represents the power received from the $k$ th user. When assuming that the elements of $\left\{\boldsymbol{s}_{k}\right\}$ are iid circularly symmetric complex random variables with zero mean and variance $1 / \mathcal{N}$, Theorem 3.1 in [7] shows that $\gamma_{k}$ converges to a deterministic value $\gamma_{k}^{*}$ as the number of users $K$ and the length $\mathcal{N}$ of spreading sequences tend to infinity, while their ratio of $\beta=K / \mathcal{N}$ is fixed. Furthermore, $\gamma_{k}^{*}$ is given by [7]

$$
\gamma_{k}^{*}=\frac{P_{k}}{\sigma^{2}+\beta E_{P}\left[I\left(P, P_{k}, \gamma_{k}^{*}\right)\right]}
$$

where

$$
I\left(P, P_{k}, \gamma_{k}^{*}\right) \equiv \frac{P P_{k}}{P_{k}+P \gamma_{k}^{*}}
$$

and in (15) and (16) $P$ is a random variable taking into account of the distribution of a user's received power.

Specifically, for our considered TF/MC DS-CDMA systems using ideal power-control, we have $P_{1}=P_{2}=\cdots=P_{K}=P_{0}$ and $\operatorname{Pr}\left(P=P_{0}\right)=1$. In this case, a simple solution to (15) can be obtained, which is [7]

$$
\gamma_{k}^{*}=\frac{(1-\beta) P_{0}}{2 \sigma^{2}}-\frac{1}{2}+\sqrt{\frac{(1-\beta)^{2} P_{0}^{2}}{4 \sigma^{4}}+\frac{(1+\beta) P_{0}}{2 \sigma^{2}}+\frac{1}{4}}
$$

\section{A. GAM-Assisted MMSE-MUD}

When comparing (12) and (14), we can find that for a given value of $\gamma_{t}$ the asymptotic SINR of $\gamma_{g a m}$, i.e. $\gamma_{g a m}^{*}$, can be obtained from (17). From (9) we can see that $\gamma_{t}^{*}$ of the asymptotic $\gamma_{t}$ can also be directly obtained from (17), which is given by

$$
\gamma_{t}^{*}=\frac{\left(1-\beta_{t}\right)}{2 \sigma^{2}}-\frac{1}{2}+\sqrt{\frac{\left(1-\beta_{t}\right)^{2}}{4 \sigma^{4}}+\frac{\left(1+\beta_{t}\right)}{2 \sigma^{2}}+\frac{1}{4}}
$$

Consequently, when replacing $\gamma_{t}$ in (12) by $\gamma_{t}^{*}$, the asymptotic SINR of the GAM-assisted MMSE-MUD can be derived, which is expressed as

$$
\begin{aligned}
\gamma_{a p p}^{*}= & \frac{\left(1-\beta_{f}\right) \gamma_{t}^{*}}{2}-\frac{1}{2} \\
& +\sqrt{\frac{\left(1-\beta_{f}\right)^{2} \gamma_{t}^{* 2}}{4}+\frac{\left(1+\beta_{f}\right) \gamma_{t}^{*}}{2}+\frac{1}{4}}
\end{aligned}
$$

As (18) and (19) show, the asymptotic SINR of the MMSE-MUD using GAM is only depended on the T-domain system load $\beta_{t}$, Fdomain system load $\beta_{f}$ as well as the noise variance $\sigma^{2}$.

\section{B. ACM-Assisted MMSE-MUD}

In the context of the ACM-assisted MMSE-MUD, the SINR after the separate MMSE-MUD is given by (13). Expressing $\boldsymbol{C}_{f} \boldsymbol{C}_{f}^{H}=$ $\boldsymbol{C}_{f 1} \boldsymbol{C}_{f 1}^{H}+\boldsymbol{c}_{f}^{(1)} \boldsymbol{c}_{f}^{(1) H}$ and applying the Sherman-Morrison formula, (13) can be written as

$$
\gamma_{a c m}=\left|q_{11}\right|^{2} \frac{\left(\boldsymbol{c}_{f}^{(1)}\right)^{H} \boldsymbol{B}^{-1} \boldsymbol{c}_{f}^{(1)}}{1+\sum_{n=2}^{N}\left|q_{1 n}\right|^{2}\left(\boldsymbol{c}_{f}^{(1)}\right)^{H} \boldsymbol{B}^{-1} \boldsymbol{c}_{f}^{(1)}}
$$

where $\boldsymbol{B}$ is defined as

$$
\boldsymbol{B}=\left[\sum_{n=1}^{N}\left|q_{1 n}\right|^{2} \boldsymbol{C}_{f 1} \boldsymbol{C}_{f 1}^{H}+\sum_{n=1}^{N_{t}}\left|p_{1 n}\right|^{2} \sigma^{2} \boldsymbol{I}\right]
$$

As shown in (20) and (21), in order to derive the asymptotic SINR of $\gamma_{a c m}^{*}$, the terms of $\sum_{n=1}^{N}\left|q_{1 n}\right|^{2}, \sum_{n=1}^{N_{t}}\left|p_{1 n}\right|^{2}$ and $\left|q_{11}\right|^{2}$ are required to be obtained first. Let us first proof the following theorem.

Theorem 1: Let $\boldsymbol{H}$ be an $(\mathcal{N} \times K)$ matrix with entries being iid complex random variables distributed with zero-mean and a common variance $1 / \mathcal{N}$. Let $\boldsymbol{R}=\boldsymbol{H}^{H} \boldsymbol{H}$ be the correlation matrix of $\boldsymbol{H}$. Then, for a fixed value of $\beta=K / \mathcal{N}$, the diagonal entries of $\boldsymbol{R}^{i}$ become equal, when $\mathcal{N}, K \rightarrow \infty$.

Proof: Let $\boldsymbol{h}_{n}$ be the $n$th column of $\boldsymbol{H}$ and $\boldsymbol{H}_{n}$ be a matrix formed from $\boldsymbol{H}$ by removing its $n$th column. Furthermore, let $\boldsymbol{R}_{H}=$ $\boldsymbol{H} \boldsymbol{H}^{H}$ be the autocorrelation matrix of $\boldsymbol{H}$. Then, it can be shown that the $(n, n)$ th entry of $\boldsymbol{R}^{i+1}$ is given by $\boldsymbol{R}^{i+1}(n, n)=\boldsymbol{h}_{n}^{H} \boldsymbol{R}_{H}^{i} \boldsymbol{h}_{n}$. Observing that $\boldsymbol{R}_{H}=\boldsymbol{H}_{n} \boldsymbol{H}_{n}^{H}+\boldsymbol{h}_{n} \boldsymbol{h}_{n}^{H}$, we have

$$
\begin{gathered}
\boldsymbol{R}^{i+1}(n, n)=\boldsymbol{h}_{n}^{H} \boldsymbol{R}_{H}^{i-1}\left(\boldsymbol{H}_{n} \boldsymbol{H}_{n}^{H}+\boldsymbol{h}_{n} \boldsymbol{h}_{n}^{H}\right) \boldsymbol{h}_{n} \\
\quad=\boldsymbol{h}_{n}^{H} \boldsymbol{R}_{H}^{i-1}\left(\boldsymbol{H}_{n} \boldsymbol{H}_{n}^{H}\right) \boldsymbol{h}_{n}+\boldsymbol{R}^{i}(n, n) \boldsymbol{h}_{n}^{H} \boldsymbol{h}_{n}
\end{gathered}
$$

Furthermore, in the above equation

$$
\begin{aligned}
\boldsymbol{h}_{n}^{H} \boldsymbol{R}_{H}^{i-1} & \left(\boldsymbol{H}_{n} \boldsymbol{H}_{n}^{H}\right) \boldsymbol{h}_{n}=\boldsymbol{h}_{n}^{H} \boldsymbol{R}_{H}^{i-2}\left(\boldsymbol{H}_{n} \boldsymbol{H}_{n}^{H}\right)^{2} \boldsymbol{h}_{n} \\
& +\boldsymbol{R}^{i-1}(n, n) \boldsymbol{h}_{n}^{H}\left(\boldsymbol{H}_{n} \boldsymbol{H}_{n}^{H}\right) \boldsymbol{h}_{n}
\end{aligned}
$$

When we continue to expand the term $\boldsymbol{h}_{n}^{H} \boldsymbol{R}_{H}^{i-j}\left(\boldsymbol{H}_{n} \boldsymbol{H}_{n}^{H}\right)^{j} \boldsymbol{h}_{n}$ in the above equation until $(i-j)=0$ and substitute the related results into (22), we finally obtain a recursive formula for $R^{i+1}(n, n)$, which is given by

$$
\boldsymbol{R}^{i+1}(n, n)=\sum_{j=0}^{i} \boldsymbol{R}^{i-j}(n, n) \boldsymbol{h}_{n}^{H}\left(\boldsymbol{H}_{n} \boldsymbol{H}_{n}^{H}\right)^{j} \boldsymbol{h}_{n}
$$


According to Lemma 1 and its corollary in [9], the relations of

$$
\boldsymbol{q}^{H} \boldsymbol{A} \boldsymbol{q}-\frac{1}{\mathcal{N}} \operatorname{Tr}(\boldsymbol{A}) \rightarrow 0 \text { and } \boldsymbol{q}^{H} \boldsymbol{A} \boldsymbol{r} \rightarrow 0
$$

hold almost surely as $\mathcal{N} \rightarrow \infty$, provided that $\boldsymbol{A}, \boldsymbol{q}$ and $\boldsymbol{r}$ are independent arguments. In (25) $\operatorname{Tr}(\boldsymbol{A})$ denotes the trace of matrix $\boldsymbol{A}$. Hence, when $\mathcal{N} \rightarrow \infty$, in (24) we have

$$
\boldsymbol{h}_{n}^{H}\left(\boldsymbol{H}_{n} \boldsymbol{H}_{n}^{H}\right)^{j} \boldsymbol{h}_{n}=\frac{1}{\mathcal{N}} \operatorname{Tr}\left(\left[\boldsymbol{H}_{n} \boldsymbol{H}_{n}^{H}\right]^{j}\right)
$$

with probability one. Furthermore, according to the Marcenko-Pastur Law [6], it can be readily shown that the term $\boldsymbol{h}_{n}^{H}\left(\boldsymbol{H}_{n} \boldsymbol{H}_{n}^{H}\right)^{j} \boldsymbol{h}_{n}$ is not depended on $n$. Based on this property, we can see that $\boldsymbol{R}(n, n)=$ $\boldsymbol{h}_{n}^{H} \boldsymbol{h}_{n}$ is not depended on $n$, implying that the diagonal entries of $\boldsymbol{R}$ are equal. Finally, with the aid of (26) and the recursive equation of (24), we can find that $\boldsymbol{R}^{i}(n, n)$ is also not depended on $n$, explaining that the diagonal entries of $\boldsymbol{R}^{i}$ are equal.

With the aid of the above theorem, we can now find the terms $\sum_{n=1}^{N}\left|q_{1 n}\right|^{2}, \sum_{n=1}^{N_{t}}\left|p_{1 n}\right|^{2}$ and $\left|q_{11}\right|^{2}$ as seen in (20). After some examining, we can find that $\sum_{n=1}^{N}\left|q_{1 n}\right|^{2}, \sum_{n=1}^{N_{t}}\left|p_{1 n}\right|^{2}$ and $q_{11}$ are respectively the $(1,1)$ th element of $\boldsymbol{Q} Q^{\boldsymbol{H}}, \boldsymbol{P} \boldsymbol{P}^{H}$ and $\boldsymbol{Q}$. Explicitly, the matrices $Q Q^{\boldsymbol{H}}, \boldsymbol{P} \boldsymbol{P}^{H}$ and $\boldsymbol{Q}$ are functions of $\boldsymbol{R}_{t}$, which can be represented as the infinite polynomials of $\boldsymbol{R}_{t}$, after applying expansion

$$
\left(\boldsymbol{R}_{t}+\sigma^{2} \boldsymbol{I}\right)^{-1}=\frac{1}{\sigma^{2}}\left(\boldsymbol{I}-\frac{1}{\sigma^{2}} \boldsymbol{R}_{t}+\frac{1}{\sigma^{4}} \boldsymbol{R}_{t}^{2}-\cdots\right)
$$

Therefore, with the aid of Theorem 3.1, we finally have for $i=$ $1,2, \ldots, N$

$$
\begin{aligned}
q_{i i} & =\frac{1}{N} \operatorname{Tr}(\boldsymbol{Q}) \\
\sum_{n=1}^{N}\left|q_{i n}\right|^{2} & =\frac{1}{N} \operatorname{Tr}\left(\boldsymbol{Q} \boldsymbol{Q}^{H}\right) \\
\sum_{n=1}^{N_{t}}\left|p_{i n}\right|^{2} & =\frac{1}{N} \operatorname{Tr}\left(\boldsymbol{P} \boldsymbol{P}^{H}\right)
\end{aligned}
$$

Furthermore, when applying the Marĉenko-Pastur Law [6], we have

$$
\begin{aligned}
q_{i i} & =\frac{1}{2 \pi \beta_{t}} \int_{a}^{b} \frac{\sqrt{(x-a)(b-x)}}{x+\sigma^{2}} d x \\
\sum_{n=1}^{N}\left|q_{i n}\right|^{2} & =\frac{1}{2 \pi \beta_{t}} \int_{a}^{b} \frac{\sqrt{(x-a)(b-x)}}{\left(x+\sigma^{2}\right)^{2}} d x \\
\sum_{n=1}^{N_{t}}\left|p_{i n}\right|^{2} & =\frac{1}{2 \pi \beta_{t}} \int_{a}^{b} \frac{\sqrt{(x-a)(b-x)}}{\left(x+\sigma^{2}\right)^{2}} d x
\end{aligned}
$$

where $a=\left(1-\sqrt{\beta_{t}}\right)^{2}$ and $b=\left(1+\sqrt{\beta_{t}}\right)^{2}$

Finally, with the results in (29), the asymptotic SINR of the ACMassisted MMSE-MUD can be evaluated in the same ways as for the GAM-assisted MMSE-MUD considered in Section III-A.

\section{PERFormance Results}

In this section we provide a few illustrations to show in what scenarios the asymptotic SINR expressions derived may be employed to evaluate the SINR performance of the TF/MC DS-CDMA systems using separate MMSE-MUD in the T- and F-domain. Furthermore, we compare the asymptotic SINR performance of the TF/MC DS-CDMA systems using GAM- and ACM-assisted MMSE-MUDs.

Figs. 2 and 3 show the asymptotic SINR as well as the SINR of several specific TF/MC DS-CDMA systems using different sets of Tdomain and F-domain spreading factors. In Fig. 2 the GAM-assisted separate MMSE-MUD is employed, while in Fig. 3 the ACM-assisted MMSE-MUD is employed. As the results in Figs. 2 and 3 illustrated, the SINR achieved by the separate MMSE-MUD converges to the

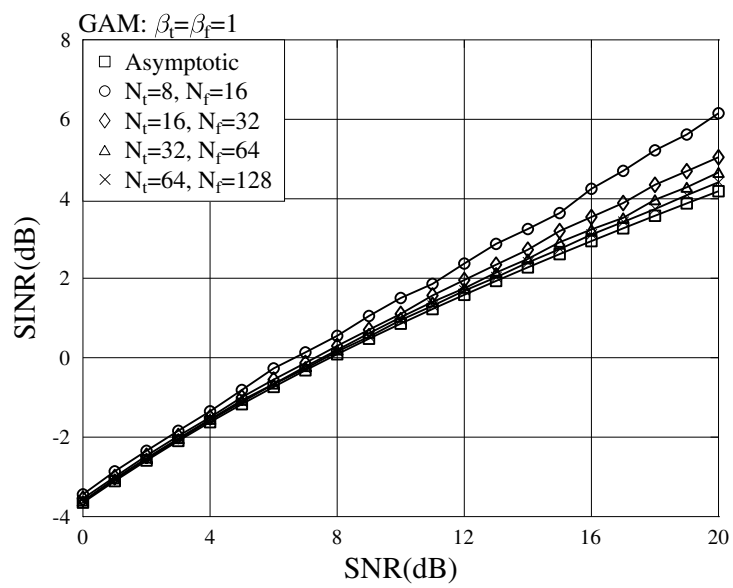

Fig. 2. Asymptotic SINR performance of the TF/MC DS-CDMA systems employing the GAM-assisted separate MMSE-MUD, when the T-domain and F-domain load factors are $\beta_{t}=\beta_{f}=1$.

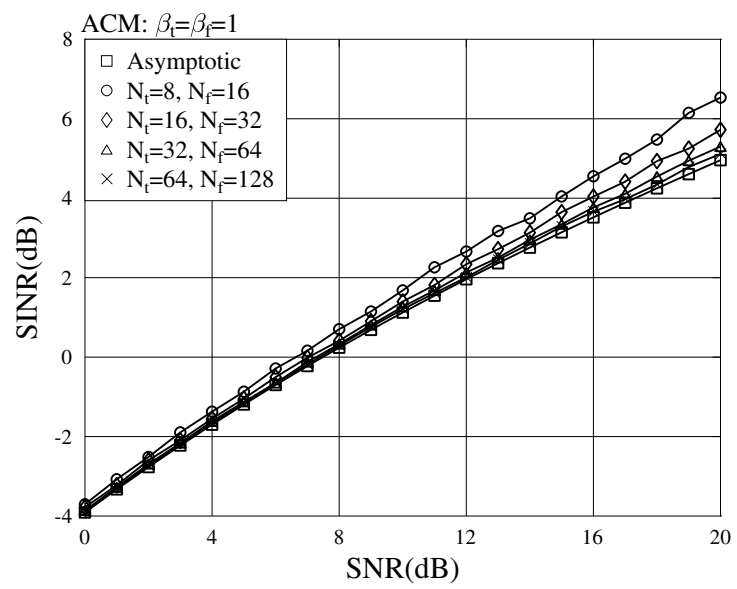

Fig. 3. Asymptotic SINR performance of the TF/MC DS-CDMA systems employing the ACM-assisted separate MMSE-MUD, when the T-domain and F-domain load factors are $\beta_{t}=\beta_{f}=1$.

asymptotic SINR, as the T-domain spreading factor $N_{t}$ and the Fdomain spreading factor $N_{f}$ increase. At low SNR region, such as lower than $12 \mathrm{~dB}$, the asymptotic SINR provides a good measure of the MMSE-MUD's achievable SINR, when $N_{t} \geq 16$ and $N_{f} \geq 32$. If the SNR is relatively high, such as higher than $16 \mathrm{~dB}$, the asymptotic SINR provides a very close measure of the MMSE-MUD's achievable SINR, provided that $N_{t} \geq 32$ and $N_{f} \geq 64$. Hence, when the T-domain and F-domain spreading factors are sufficiently high, the asymptotic SINR is generally capable of providing an very accurate measure of the SINR achieved by the separate MMSE-MUD.

When comparing the results in Fig. 2 with the corresponding results in Fig. 3 we can find that for each set of $\left(N_{t}, N_{f}\right)$ the SINR performance in Fig. 3 is slightly better than that in Fig. 2, implying that the ACM-assisted separate MMSE-MUD outperforms the GAM-assisted separate MMSE-MUD. This is because with the ACMassisted separate MMSE-MUD the F-domain MMSE-MUD tries to further suppress the residual MUI after the T-domain MMSE-MUD. By contrast, for the GAM-assisted separate MMSE-MUD, the Fdomain MMSE-MUD treats the T-domain MMSE-MUD's residual MUI as Gaussian noise. 


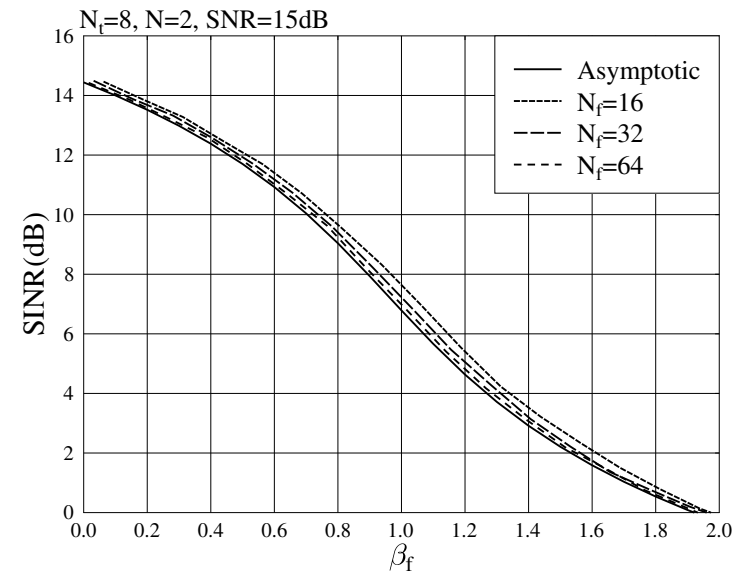

Fig. 4. Asymptotic SINR performance of the TF/MC DS-CDMA systems supporting two user groups and using the ACM-assisted separate MMSEMUD, when various F-domain load factors are considered.

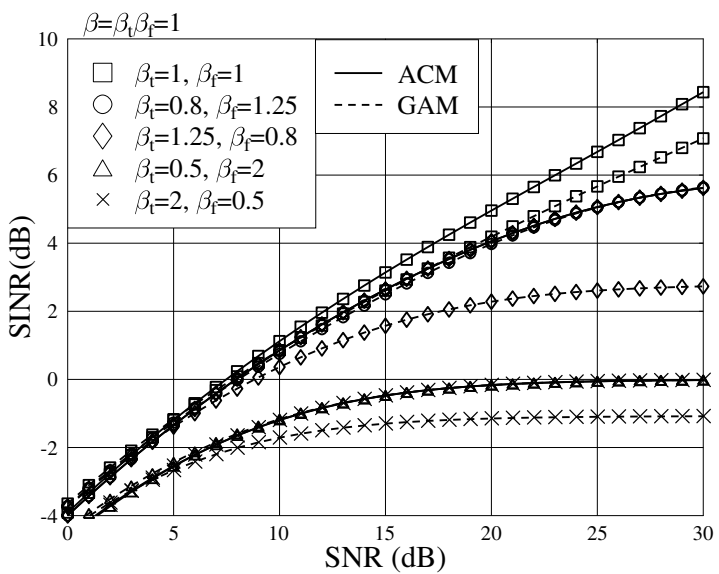

Fig. 5. Asymptotic SINR performance of TF/MC DS-CDMA systems having different F-domain and T-domain load factors, while the total system load factor retains $\beta=1$.

Fig. 4 shows the asymptotic as well as the simulated SINR performance versus the F-domain load factor for the TF/MC DS-CDMA systems supporting two user groups, when the ACM-assisted MMSEMUD is applied. Explicitly, the asymptotic SINR provides good approximation for the SINR achieved by the ACM-assisted MMSEMUD for all the cases considered. As seen in Fig. 4, for all the cases the differences between the ACM-assisted MMSE-MUD's achievable SINR and the corresponding asymptotic SINR is less than one decibel.

Finally, in Fig. 5 we compare the asymptotic SINR performance of the TF/MC DS-CDMA systems employing the GAM- or ACMassisted MMSE-MUD, when different T-domain and F-domain load factors are used while the total system load factor retains constant $\beta=1$. Note that, in Fig. 5 the SINR performance of the cases ACM $\left(\beta_{t}=0.8, \beta=1.25\right)$, ACM $\left(\beta_{t}=1.25, \beta=0.8\right)$ and GAM $\left(\beta_{t}=0.8, \beta=1.25\right)$ are the same; The SINR performance of the cases $\operatorname{ACM}\left(\beta_{t}=0.5, \beta=2\right), \operatorname{ACM}\left(\beta_{t}=2, \beta=0.5\right)$ and GAM $\left(\beta_{t}=0.5, \beta=2\right)$ are also the same. Except the above cases, we can see for all the other cases the ACM-assisted MMSE-MUD outperforms the GAM-assisted MMSE-MUD. The reason for the GAM-assisted MMSE-MUD under $\left(\beta_{t}=0.8, \beta=2\right)$ or $\left(\beta_{t}=0.5, \beta=2\right)$ achieving the same SINR performance as the ACM-assisted MMSEMUD under the same case is that the T-domain load is light. Hence, the T-domain MMSE-MUD is capable of efficiently suppressing the MUI, making the residual MUI ignorable. Consequently, both the GAMand ACM-assisted MMSE-MUDs achieve a similar asymptotic SINR performance.

\section{Conclusions}

With the aid of random matrix theory we have investigated the asymptotic SINR performance of the TF/MC DS-CDMA systems using separate MMSE-MUD, when communicating over AWGN channels. Two types of separate MMSE-MUDs, namely the GAM- and ACM-assisted MMSE-MUDs, have been considered, which treat the T-domain MMSE-MUD's residual MUI as Gaussian noise or interference that is further suppressed by the F-domain MMSE-MUD. A range of closed-form expressions facilitating to evaluate have been derived. Simulation results show that in most cases the SINR values of the TF/MC DS-CDMA systems using separate MMSE-MUD are close to that evaluated by the closed-form expressions. Furthermore, our studies demonstrate that the ACM-assisted MMSE-MUD usually outperforms the GAM-assisted MMSE-MUD when considering their achievable SINR performance. Given a constant system load factor, for both the MMSE-MUDs, the TF/MC DS-CDMA systems with the same T-domain and F-domain load factors usually achieve the best SINR performance.

\section{ACKNOWLEDGMENT}

The authors would like to acknowledge with thanks the financial assistance from the Natural Science Foundation of China under the grant No. 60921001 and the EPSRC of the UK.

\section{REFERENCES}

[1] J. Wang and L.B. Milstein, "Multicarrier CDMA overlay for ultra- wideband communications," IEEE Trans on Commun., vol. 52, no. 10, pp. 1664-1669, Oct. 2004.

[2] Y. Zhou, J. Wang and M. Sawahashi, "Downlink transmission of broadband OFCDM Systems-part I: hybrid detection," IEEE Trans. on Communications, Vol. 53, No. 4, pp. 718 - 729, Apr. 2005.

[3] L.-L. Yang, Multicarrier Communications. Chichester, United Kingdom: John Wiley, 2009.

[4] L.-L. Yang, W. Hua, and L. Hanzo, "Multiuser detection assisted time- and frequency-domain spread multicarrier code-division multipleaccess," IEEE Trans. on Veh. Tech., vol. 55, no. 1, pp. 397 - 405, Jan. 2006.

[5] L.-L. Yang and L.-C. Wang, "Zero-forcing and minimum mean-square error multiuser detection in generalized multicarrier DS-CDMA systems for cognitive radio," EURASIP J. on Wireless Commun. and Net., vol. 2008, no. 5, Article ID 541410, doi:10.1155/2008/541410, 2008.

[6] A. M. Tulino and S. Verdu, Random Matrix Theory and Wireless Communications. Amsterdam, The Netherlands: Now Publishers, 2004.

[7] D. N. C. Tse and S. V. Hanly, "Linear multiuser receiver: effective interference, effective bandwidth and user capacity," IEEE Trans. on Infor. Theory, vol. 45, no. 2, pp. 641 - 657, Mar. 1999.

[8] S. Verdu and S. Shamai, "Spectral efficiency of CDMA with random spreading," IEEE Trans. on Infor. Theory, vol. 45, no. 2, pp. $622-640$, Mar. 1999.

[9] J. Evans and D. N. C. Tse, "Large system performance of linear multiuser receivers in multipath fading channels," IEEE Trans on Infor. Theory, vol. 46 , no. 6 , pp. 2059 - 2078, Sept. 2000

[10] J. Wang and J. Chen, "Performance of wideband CDMA with complex spreading and imperfect channel estimation," IEEE J. on Select. Areas in Commun., vol. 19, pp. 152-163, Jan. 2001.

[11] A. M. Tulino, L. Li, and S. Verdu, "Spectral efficiency of multicarrier CDMA," IEEE Trans. on Infor. Theory, vol. 51, no. 2, pp. $479-505$, Feb. 2005.

[12] C.-N. Chuah, D. N. C. Tse, J. M. Kahn, and R. A. Valenzuela, "Capacity scaling in MIMO wireless systems under correlated fading," IEEE Trans. on Infor. Theory, vol. 48, no. 3, pp. 637 - 650, Mar. 2002.

[13] P. Pan, L.-L. Yang, and Y. Zhang, "Spectral-efficiency of time-frequencydomain spread multicarrier DS-CDMA in frequency-selective Nakagami$m$ fading channels," in IEEE VTC2008-Fall. IEEE, Sept. 2008.

[14] U. Madhow and M. L. Honig, "MMSE interference suppression for directsequence spread-spectrum CDMA," IEEE Trans. on Commun., vol. 42, no. 12 , pp. $3178-3188$, Dec. 1994. 